\title{
Cardiac Resynchronization Therapy Leads to Improvements in Handgrip Strength
}

\author{
David R. Warriner ${ }^{\mathrm{a}, \mathrm{b}, \mathrm{d}}$, Patricia Lawford ${ }^{\mathrm{a}}$, Paul J. Sheridan ${ }^{\mathrm{c}}$
}

\begin{abstract}
Background: A reduction in skeletal muscle performance measured by handgrip strength is common in heart failure. No trial has investigated the role of cardiac resynchronization therapy, which leads to improvements in cardiac performance, on the function of skeletal muscle in patients with heart failure.
\end{abstract}

Methods: Nineteen patients were recruited, 18 male, age $69 \pm 8$ years, New York Heart Association class II-IV, QRS duration $173 \pm$ $21 \mathrm{~ms}$ and left ventricular ejection fraction $26 \pm 8 \%$. Handgrip strength was measured at baseline before, and 6 and 12 months, following cardiac resynchronization therapy. Response was assessed using quality of life questionnaire, 6-minute walk distance, left ventricular enddiastolic volume, and cardiopulmonary exercise testing at the same time points.

Results: Fourteen patients were identified as responders, demonstrating significant improvements in all four markers of response. There was no significant difference at baseline in left or right handgrip strength between responders and non-responders. Compared to baseline, handgrip strength significantly increased in responders during follow-up, left $(34.4 \pm 11.4$ to $40.3 \pm 11.3 \mathrm{kgf}, \mathrm{P}<0.001)$ and right $(35.7 \pm 12.5$ to $42.2 \pm 11.5 \mathrm{kgf}, \mathrm{P}<0.001)$ at 12 months. No such improvement was seen in non-responders.

Conclusions: This study demonstrates that positive response to cardiac resynchronization therapy is associated with significant gains in handgrip strength, suggesting that cardiac resynchronization therapy may indirectly lead to secondary gains in skeletal muscle function.

Keywords: Cardiac resynchronization therapy; Heart failure; Hand-

Manuscript accepted for publication June 09, 2016

${ }^{a}$ Medical Physics Group, Department of Cardiovascular Science, The Medical School, University of Sheffield, Sheffield, S10 2TN, UK

bDepartment of Cardiology, Northern General Hospital, Sheffield Teaching Hospitals, Sheffield, S5 7AU, UK

'Department of Cardiology, Chesterfield Royal Hospital, Chesterfield, Derbyshire, S44 5BL, UK

${ }^{\mathrm{d} C o r r e s p o n d i n g ~ A u t h o r: ~ D a v i d ~ R . ~ W a r r i n e r, ~ M e d i c a l ~ P h y s i c s ~ G r o u p, ~ D e p a r t-~}$ ment of Cardiovascular Science, The Medical School, Beech Hill Road, Sheffield, S10 2RX, UK. Email: d.r.warriner@sheffield.ac.uk

doi: http://dx.doi.org/10.14740/cr475w grip strength

\section{Introduction}

Heart failure (HF) is not a single organ disease but rather a multifaceted syndrome, which can result in psychological, musculoskeletal, hematological, pulmonary, endocrine, endothelial, renal and hepatic impairment [1]. The muscle hypothesis of HF proposed that "exercise performance in HF patients is predominantly limited by skeletal muscle and less by the performance of cardiac muscle" [2]. Handgrip strength (HGS) is the maximum force that can be generated by the hand and forearm musculature during a single isometric contraction and as such is a measure of skeletal muscle performance. HGS correlates well with overall body strength, is used as a marker of exercise capacity, general health and nutritional status and deteriorates with aging and chronic disease. In HF, HGS can be used to stratify patients according to functional class, peak $\mathrm{VO}_{2}$ and is an independent predictor of survival [3-7]. Previous clinical trials have shown pharmacotherapies commonly used in HF, such as angiotensin converting enzyme (ACE) inhibitors, to preserve lean body weight but not affect skeletal muscle function $[8,9]$.

Cardiac resynchronization therapy (CRT) is a widely used treatment in HF; it improves the hearts pumping efficiency by restoring synchronous contraction of the atria and ventricles. Benefits include improved quality of life and exercise capacity and reduced hospitalization and mortality [10]. Despite meeting strict implantation criteria, only two-thirds of patients respond to this complex device therapy and it still remains unclear as to why this is the case [11].

To date, no trial has investigated the role of CRT influencing skeletal muscle performance, such as measuring HGS, presenting a means of differentiating between responders and non-responders, at baseline and during follow-up. If HGS, a simple, inexpensive and reliable test could be used to differentiate likely responders from non-responders at baseline preCRT implantation, then this would be invaluable to clinicians, patient groups and researchers alike. Furthermore, any differences in HGS as a result of CRT identified during follow-up would further refine current understanding of response to CRT and possible reasons underlying non-response. 


\section{Methods}

\section{Ethics approval and consent to participate}

All patients gave fully informed written consent to take part in the project. The project was conducted in compliance with the principles of the Declaration of Helsinki (October 2008), the principles of Good Clinical Practice (GCP) and all of the applicable regulatory requirements. The project was approved by the National Research Ethics Service (NRES) number 10/ H0802/71.

\section{Recruitment}

Patients were screened for eligibility by a physician and a dedicated clinical research fellow. Nineteen patients who received CRT based on current clinical criteria used at Sheffield Teaching Hospitals (STH) NHS Trust were recruited for this study, i.e., patients with an ejection fraction $<35 \%$, a QRSd $>120$ ms, NYHA functional class II-IV and optimal medical therapy. No patients had a history of wrist or hand arthritis and patients were examined specifically prior to starting the study.

\section{Study design}

The study was a prospective cohort study using an opportunity sample. All patients recruited to the study had their HGS assessed at baseline, 6 and at 12 months ( \pm 2 weeks) post-CRT implantation. At the same time points, they underwent echocardiography and cardiopulmonary exercise testing (CPET) to measure their peak $\mathrm{VO}_{2}$ and completed the Minnesota Living With Heart Failure Questionnaire (MLWHFQ). At 6 weeks after CRT implantation, all patients were referred for routine echocardiography-guided CRT optimization, using the iterative method to optimize atrio-ventricular delay and the aortic velocity time integral method for the inter-ventricular delay.

\section{Assessment of grip strength}

HGS was measured at baseline, 6 and 12 months using a single protocol; patients were asked to perform three maximal trials with each hand using a Jamar analogue dynamometer on the morning of testing. The patients were all positioned according to the American Society of Hand Therapists (ASHT) guidance [11]. Standardized verbal instructions and encouragement were used as recommended by Mathiowetz et al (1984) [12]. The investigator carrying out HGS measurement was blinded to markers of response to CRT. The analogue Jamar dynamometer used to measure HGS had excellent test-retest $(\mathrm{r}=0.88$ $-0.93)$ and inter-rater $(r=0.99)$ reliability [12]. Measurement of HGS was known to be sensitive to a range of factors such as patient encouragement, time of day, posture, the position of the upper limb joints (shoulder, elbow, and wrist) and even the position of the dynamometer handle itself, hence it was essen- tial to use a standardized approach [13]. There was a learning effect with using the dynamometer and in an attempt to negate this, the best of three attempts for both right and left hands was used.

\section{Assessment of response to CRT}

A positive response to CRT was defined a priori by the authors, as a significant improvement in all of four different outcome measures, a $>1 \mathrm{~mL} / \mathrm{kg} / \mathrm{min}$ increase in peak $\mathrm{VO}_{2}, a>15 \%$ reduction in left ventricular end-diastolic volume (LVEDV), a $>10 \%$ increase in 6-min walk distance (6MWD) and a $>10$ point reduction in symptoms as measured by the MLWHFQ quality of life $(\mathrm{QoL})$ questionnaire.

CPET consisted of a ramp protocol on a static bicycle ergometer to measure peak $\mathrm{VO}_{2}$ supervised by a senior pulmonary physiologist. Peak $\mathrm{VO}_{2}$ was the highest measure of $\mathrm{VO}_{2}$ attained, but not necessarily that, which would be ultimately attainable and had been shown to improve significantly in responders to CRT.

LVEDV was calculated using the modified Simpson's rule of stack discs using apical two- and four-chamber views by two-dimensional echocardiography General Electric (GE Healthcare, Princeton, NJ) Vivid 7 ultrasound machine, assessed by senior echocardiographers.

The 6-min walk test (6MWT) measured the distance walked at a normal pace on a flat, hard, even surface in $6 \mathrm{~min}$, the 6MWD.

The MLWHFQ was a validated and well-tested questionnaire assessing the impact of HF on QoL, and specifically it asked questions on domains relating to both psychological and physiological factors, including questions on the side-effects of treatments, hospital admissions, symptoms, mood, sex-life and appetite. The investigators administered the questionnaire.

\section{Statistical analysis}

Statistical analysis was performed using SPSS software (version 21.0, SPSS Inc., Chicago, IL, USA). Assessment of data for normality was carried out using both visual assessment of the data and Shapiro-Wilks test. Categorical data were analyzed using a two-tailed Fisher's exact test. Parametric data are given in terms of the mean $\pm \mathrm{SD}$. Comparison of data between groups at baseline was performed using an unpaired two-tailed Student's $t$-test. Comparison of data within groups during follow was performed using one-way ANOVA with repeated measures. Correlations were analyzed with the Pearson's correlation coefficient. P values of $<0.05$ were considered significant.

\section{Results}

Nineteen patients were included in the study. The baseline patient characteristics are shown in Table 1 . As can be seen, all patients had similarly impaired LV function at baseline and a 
Table 1. Baseline Characteristics of Responders and Non-Responders Pre-CRT Implantation

\begin{tabular}{|c|c|c|c|c|c|}
\hline & \multicolumn{2}{|c|}{ Responders $(\mathrm{N}=14)$} & \multicolumn{2}{|c|}{ Non-responders $(\mathrm{N}=\mathbf{5})$} & \multirow[t]{2}{*}{ P value } \\
\hline \multicolumn{5}{|l|}{ Demographics } & \\
\hline Age (years) & 68.4 & 9.8 & 71.8 & 4.2 & 0.53 \\
\hline Gender (male) & 13 & $93 \%$ & 5 & $100 \%$ & $0.99 *$ \\
\hline Handedness (right) & 14 & $100 \%$ & 5 & $100 \%$ & $0.99 *$ \\
\hline Body mass index $\left(\mathrm{kg} / \mathrm{m}^{2}\right)$ & 29.6 & 3.98 & 26.5 & 4.41 & 0.18 \\
\hline Ischemic heart disease & 8 & $57 \%$ & 3 & $60 \%$ & $0.99 *$ \\
\hline Hypercholesterolemia & 10 & $72 \%$ & 4 & $80 \%$ & $0.99 *$ \\
\hline Hypertension & 7 & $50 \%$ & 2 & $40 \%$ & $0.99 *$ \\
\hline Chronic kidney disease & 4 & $29 \%$ & 4 & $80 \%$ & $0.11 *$ \\
\hline Diabetes & 5 & $36 \%$ & 1 & $20 \%$ & $0.99 *$ \\
\hline \multicolumn{6}{|l|}{ ECG/echocardiography } \\
\hline QRSd (ms) & 163.0 & 22.8 & 155.5 & 20.9 & 0.55 \\
\hline $\mathrm{EF}(\%)$ & 25.6 & 8.0 & 25.8 & 8.5 & 0.44 \\
\hline \multicolumn{6}{|l|}{ Medications } \\
\hline ACEI/ARB & 13 & $93 \%$ & 5 & $100 \%$ & $0.99 *$ \\
\hline Beta-blocker & 12 & $86 \%$ & 5 & $100 \%$ & $0.99 *$ \\
\hline Loop diuretic & 14 & $100 \%$ & 5 & $100 \%$ & $0.99 *$ \\
\hline Spironolactone & 9 & $64 \%$ & 4 & $80 \%$ & $0.99 *$ \\
\hline \multicolumn{6}{|l|}{ Markers of response } \\
\hline 6MWD (m) & 374.0 & 112.8 & 337.0 & 144.7 & 0.23 \\
\hline LVEDV (mL) & 201.5 & 72.5 & 159.0 & 80.8 & 0.37 \\
\hline MLWHFQ & 44.4 & 22.9 & 52.8 & 22.7 & 0.33 \\
\hline Peak $\mathrm{VO}_{2}(\mathrm{~mL} / \mathrm{kg} / \mathrm{min})$ & 12.5 & 1.6 & 13.9 & 2.7 & 0.25 \\
\hline \multicolumn{6}{|l|}{ Hand grip strength (kgf) } \\
\hline Left & 34.4 & 11.4 & 33.0 & 5.3 & 0.42 \\
\hline Right & 35.7 & 12.5 & 31.2 & 12.2 & 0.25 \\
\hline
\end{tabular}

Mean or number given in right hand column and \% or SD given in left hand column. ^Comparisons between groups analyzed using two-tailed unpaired Student's $t$-test unless categorical data $\left({ }^{*}\right)$ compared using Fisher's exact test. ACE: angiotensin converting enzyme inhibitor; ARB: angiotensin receptor blocker; CRT: cardiac resynchronization therapy; ECG: electrocardiography; EF: ejection fraction; LVEDV: left ventricular end-diastolic volume; QRSd: QRS duration.

prolonged QRS duration. Furthermore, there were no statistically significant differences between the baseline measures of response, such as LVEDV, peak $\mathrm{VO}_{2}, 6 \mathrm{MWD}$ or MLWHFQ (Table 1).

\section{Response to CRT}

A positive response to CRT in the four criteria was observed in 14 of the 19 patients after 12 months of CRT (Table 2).

A one-way ANOVA with repeated measures and Greenhouse-Geisser correction determined that peak $\mathrm{VO}_{2}(\mathrm{~F}(1.27$, $13.9)=9.26, \mathrm{P}<0.01), \operatorname{LVEDV}(\mathrm{F}(1.73,17.3)=6.73, \mathrm{P}<$ $0.01)$, MLWHFQ $(\mathrm{F}(1.97,21.76)=7.40, \mathrm{P}<0.01)$ and $6 \mathrm{MWD}$ $(\mathrm{F}(1.93,21.03)=22.67, \mathrm{P}<0.01)$ in responders was statistically different between time points during follow-up. Post hoc analysis using the Bonferroni correction demonstrated responders had statistically significant differences between baseline and 6 months and baseline and 12 months in 6MWD (Fig. 1A), peak $\mathrm{VO}_{2}$ (Fig. 1B), MLWHFQ score (Fig. 1C) and LVEDV (Fig. 1D).

Non-responders failed to demonstrate a significant difference in any of these four markers between time points according to a one-way ANOVA with repeated measures (Table 2 and Fig. 2A-D).

There was no significant difference in the workload (Watts) measured at peak $\mathrm{VO}_{2}$ at baseline between the responders and non-responders $(91.2 \pm 24.9 \mathrm{~W}$ and $93.2 \pm 18.7 \mathrm{~W}, \mathrm{P}=0.29)$, using an unpaired Student's $t$-test.

A one-way ANOVA with repeated measures that did not violate Mauchly's test of sphericity determined that workload in responders was statistically different between time points in 
Table 2. Markers of Response at 0 (Baseline), 6 and 12 Months Follow-Up in Responders and Non-Responders

\begin{tabular}{|c|c|c|c|c|c|}
\hline \multirow{2}{*}{ Markers of response } & \multirow{2}{*}{$\begin{array}{l}\text { Baseline, } \\
\text { mean } \pm \text { SD }\end{array}$} & \multirow{2}{*}{$\begin{array}{l}6 \text { months, } \\
\text { mean } \pm S D\end{array}$} & \multirow{2}{*}{$\begin{array}{l}12 \text { months, } \\
\text { mean } \pm \mathrm{SD}\end{array}$} & \multicolumn{2}{|c|}{ One-way ANOVA with repeated measures $P$ value } \\
\hline & & & & Baseline vs. 6 months & Baseline vs. 12 months \\
\hline \multicolumn{6}{|l|}{ Responders $(\mathrm{N}=14)$} \\
\hline LVEDV (mL) & $175.1 \pm 67.2$ & $157.0 \pm 77.3$ & $121.3 \pm 72.0$ & $<0.05$ & $<0.05$ \\
\hline MLWHFQ/105 & $44.4 \pm 22.9$ & $24.4 \pm 19.1$ & $24.1 \pm 21.3$ & $<0.05$ & $<0.05$ \\
\hline 6MWD $(\mathrm{m})$ & $337.0 \pm 144.7$ & $279.2 \pm 155.5$ & $279.6 \pm 113.8$ & 0.34 & \\
\hline LVEDV (mL) & $159.0 \pm 80.8$ & $172.4 \pm 126.2$ & $145.6 \pm 88.8$ & 0.63 & \\
\hline MLWHFQ/105 & $52.8 \pm 22.7$ & $37.0 \pm 20.6$ & $36.4 \pm 26.7$ & 0.31 & \\
\hline Peak $\mathrm{VO}_{2}(\mathrm{~mL} / \mathrm{kg} / \mathrm{min})$ & $13.9 \pm 2.7$ & $11.5 \pm 4.1$ & $12.8 \pm 3.9$ & 0.21 & \\
\hline
\end{tabular}

LVEDV: left ventricular end-diastolic volume; MLWHFQ: Minnesota Living With Heart Failure Questionnaire; 6MWD: 6-minute walk distance.

$(\mathrm{F}(2,22)=4.45, \mathrm{P}<0.05)$ but not non-responders $(\mathrm{F}(2,6)=$ $0.91, \mathrm{P}=0.45)$. Post hoc analysis using pairwise comparisons in responders only revealed that workload approached statistical significance between baseline and 6 months and baseline and 12 months (Fig. 3), but not 6 and 12 months (data not shown).

\section{Hand grip strength}

There were no statistically significant differences between the HGS of responders and non-responders at baseline (Table 1). There were also no statistically significant differences in HGS in responders and non-responders between the left and right hands (data not shown).

A one-way ANOVA with repeated measures and Greenhouse-Geisser correction determined mean HGS in responders was statistically different between time points in the left $(\mathrm{F}(1.77,17.71)=21.03, \mathrm{P}<0.001)$ and the right $(\mathrm{F}(1.36$, $13.58)=16.03, \mathrm{P}<0.01)$ hand. Post hoc tests using the Bonferroni correction revealed that HGS increased statistically significantly between baseline and 6 months and baseline and 12 months, but not 6 and 12 months (data not shown), in both hands (Table 3 and Fig. 4A and B).

A one-way ANOVA with repeated measures and Greenhouse-Geisser correction determined that there were no statis-
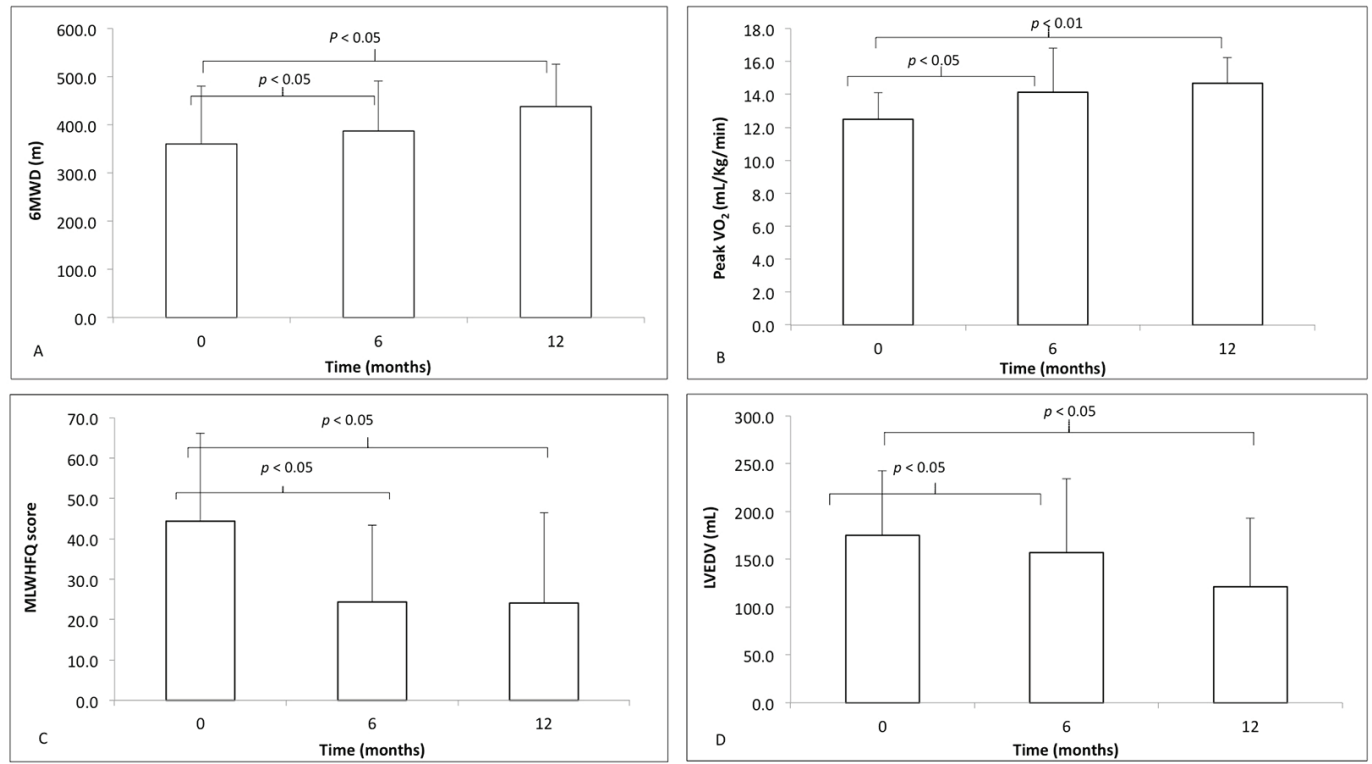

Figure 1. The markers of response to cardiac resynchronization therapy, at time zero (baseline) before implantation, 6 and 12 months following CRT implantation in responders (white): (A) 6-minute walk distance (6MWD); (B) peak $\mathrm{VO}_{2}$; (C) Minnesota Living With Heart Failure Questionnaire (MLWHFQ) score; (D) left ventricular end-diastolic volume (LVEDV). Differences in data from baseline to 6 and 12 months compared using one-way ANOVA with repeated measures. 


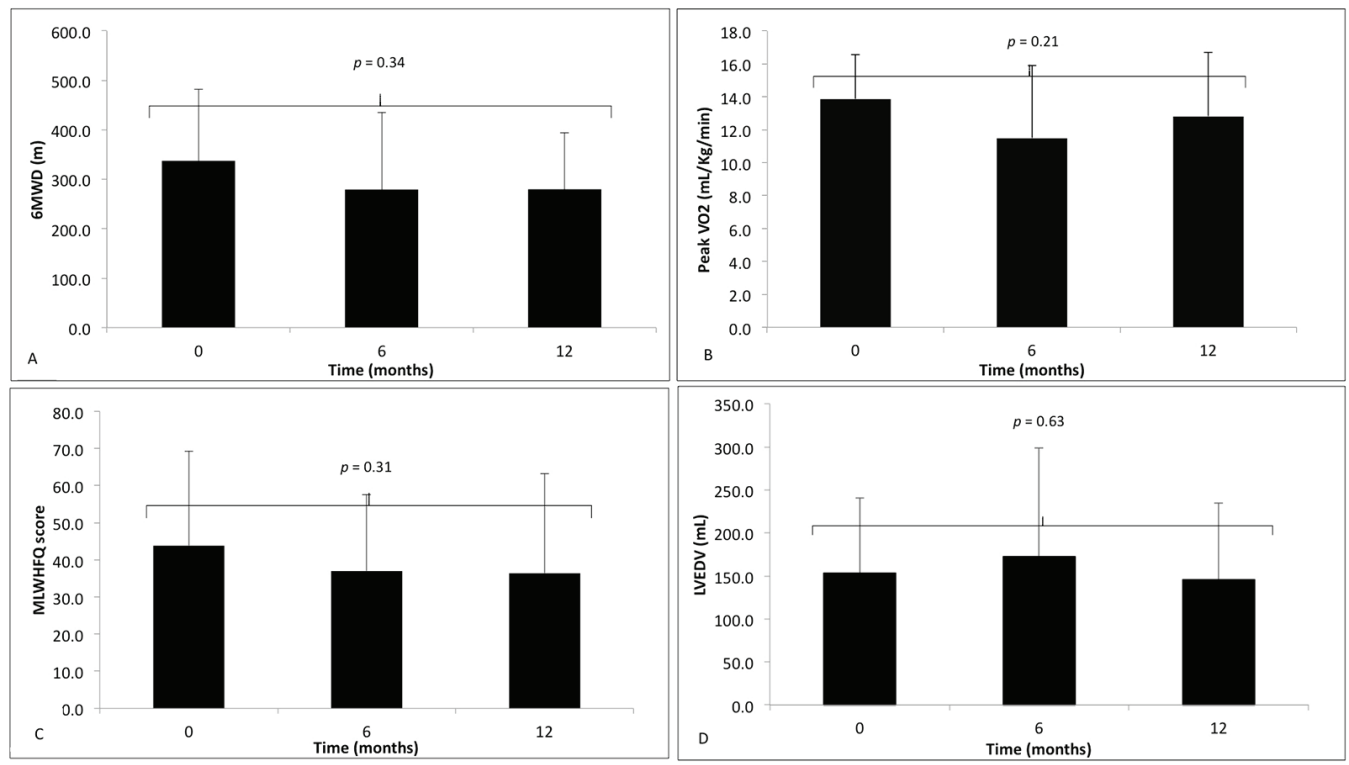

Figure 2. The markers of response to cardiac resynchronization therapy, at time zero (baseline) before implantation, 6 and 12 months following CRT implantation in non-responders (black). (A) 6-minute walk distance (6MWD); (B) peak VO ${ }_{2}$; (C) Minnesota Living With Heart Failure Questionnaire (MLWHFQ) score; (D) left ventricular end-diastolic volume (LVEDV). Data during followup compared using one-way ANOVA with repeated measures, with no significant difference.

tical differences between time points in the left $(\mathrm{F}(1.05,4.22)$ $=1.29, \mathrm{P}=0.30)$ or right $(\mathrm{F}(1.12,4.49)=0.24, \mathrm{P}=0.66)$ hand (Table 3 and Fig. $4 \mathrm{~A}$ and $\mathrm{B}$ ) of non-responders.

\section{Hand grip strength and CRT}

Pearson's correlation coefficient compared mean HGS with 6MWD during follow-up over the 12 months (Fig. 5A and $B$, respectively), which proved significant in both the left $(r=$
$0.5160, \mathrm{n}=57, \mathrm{P}<0.001)$ and right $(\mathrm{r}=0.6821, \mathrm{n}=57, \mathrm{P}<$ $0.001)$ hands.

\section{Discussion}

This study demonstrates, for the first time, a difference in HGS between responders and non-responders to CRT. At baseline, there is no significant difference in skeletal muscle function as measured by HGS. During follow-up however, there is a
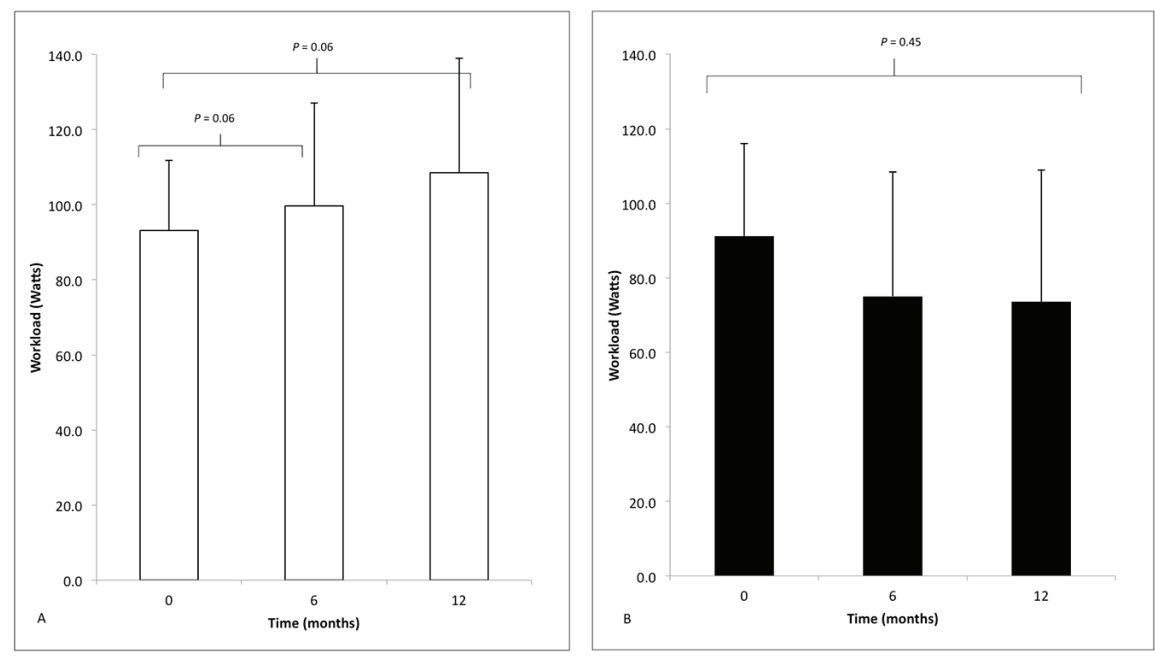

Figure 3. The peak workload (watts) measured during cardiopulmonary exercise testing in (A) responders (white) and (B) nonresponders (black) at time zero (baseline) before implantation and then 6 and 12 months following CRT, compared using one-way ANOVA with repeated measures. 
Table 3. Handgrip Strength at 0 (Baseline), 6 and 12 Months Follow-Up in Responders and Non-Responders

\begin{tabular}{|c|c|c|c|c|c|}
\hline \multirow{2}{*}{ Handgrip strength } & \multirow{2}{*}{$\begin{array}{l}\text { Baseline, } \\
\text { mean } \pm \text { SD }\end{array}$} & \multirow{2}{*}{$\begin{array}{l}6 \text { months, } \\
\text { mean } \pm \text { SD }\end{array}$} & \multirow{2}{*}{$\begin{array}{l}12 \text { months, } \\
\text { mean } \pm \text { SD }\end{array}$} & \multicolumn{2}{|c|}{ One way ANOVA with repeated measures $P$ value } \\
\hline & & & & Baseline vs. 6 months & Baseline vs. 12 months \\
\hline \multicolumn{6}{|l|}{ Responders $(\mathrm{N}=14)$} \\
\hline Right (kgf) & $35.7 \pm 12.5$ & $39.8 \pm 11.5$ & $42.2 \pm 11.2$ & $<0.01$ & $<0.001$ \\
\hline \multicolumn{6}{|c|}{ Non-responders $(\mathrm{N}=5)$} \\
\hline
\end{tabular}

kgf: kilograms of force; SD: standard deviation.

significant improvement in HGS, and whilst non-significant, there is also a decline in HGS in the non-responders. This is despite no other significant difference in physical performance between the two groups at baseline. The improvement in HGS of $17-20 \%$ is similar to gains considered significant in other metrics of response such as LVEDV, peak $\mathrm{VO}_{2}$ or MLWHFQ. These results suggest that, by improving cardiac function in responders, CRT better enables patients to perform activities of daily living and so regain strength of the skeletal muscles. Furthermore, the positive correlation with 6MWD suggests that HGS is a real-world assessment of fitness; indeed at 12 months follow-up, every $1.5 \mathrm{kgf}$ increase in HGS correlated with a $10 \mathrm{~m}$ increase in 6WMD.

It is unlikely that CRT has a direct effect on skeletal muscle other than a possible improvement of nutritive flow to the tissue, as a result of increased cardiac output [14]. To investigate possible mechanisms, skeletal muscle biopsy could be utilized to see what changes occur in skeletal muscle fiber distribution, metabolism, mitochondria and respiration, known to deteriorate in HF, improved as a consequence of CRT [15-20].

The assumption is made that gains in HGS reflect increased muscle performance throughout the body, but it would be interesting to measure this in terms of function of other major muscle groups such as the quadriceps and knee extension strength [7]. Whilst there were no significant differences at baseline in any CPET measures, there were increases in peak $\mathrm{VO}_{2}$ and peak workload but not anaerobic threshold (data not shown). Whilst the changes in workload approached significance, unlike peak $\mathrm{VO}_{2}$, this suggests that response to CRT leads to specific gains in isometric strength in the upper limbs measured by HGS, and this may also translate into more generalized improvements in skeletal muscle function, demonstrated by increase in workload in the lower limbs.

Sarcopenia is defined as the "age-associated loss of skeletal muscle mass and function" which is common in HF and independently associated with reduced exercise performance even when age, sex, functional class and co-morbidities are controlled for [21, 22]. However, in the absence of a measure of lean mass, this study investigated only differences in HGS, also known as dynapenia [23]. Whilst gains in HGS may be due to increased motor recruitment, myocyte hypertrophy may also be involved with CRT leading to a reversal of sarcopenia; measurement of lean mass by bioelectric impedance, dual energy X-ray absorptiometry or appendicular muscle volume by MRI would be needed to corroborate these findings and thus assess muscle mass and function, not just dynapenia. Clearly,
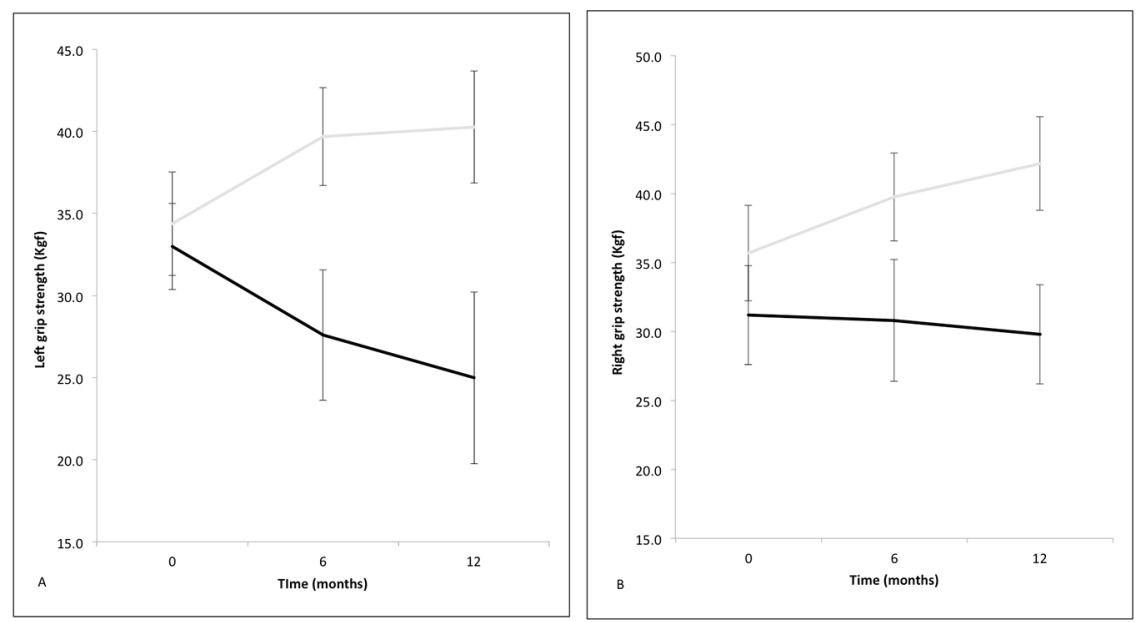

Figure 4. Mean handgrip strength in the left (A) and right hand (B) at zero (baseline), 6 and 12 months following cardiac resynchronization therapy (responders in solid gray line and non-responders in solid black line) with standard error bars. 

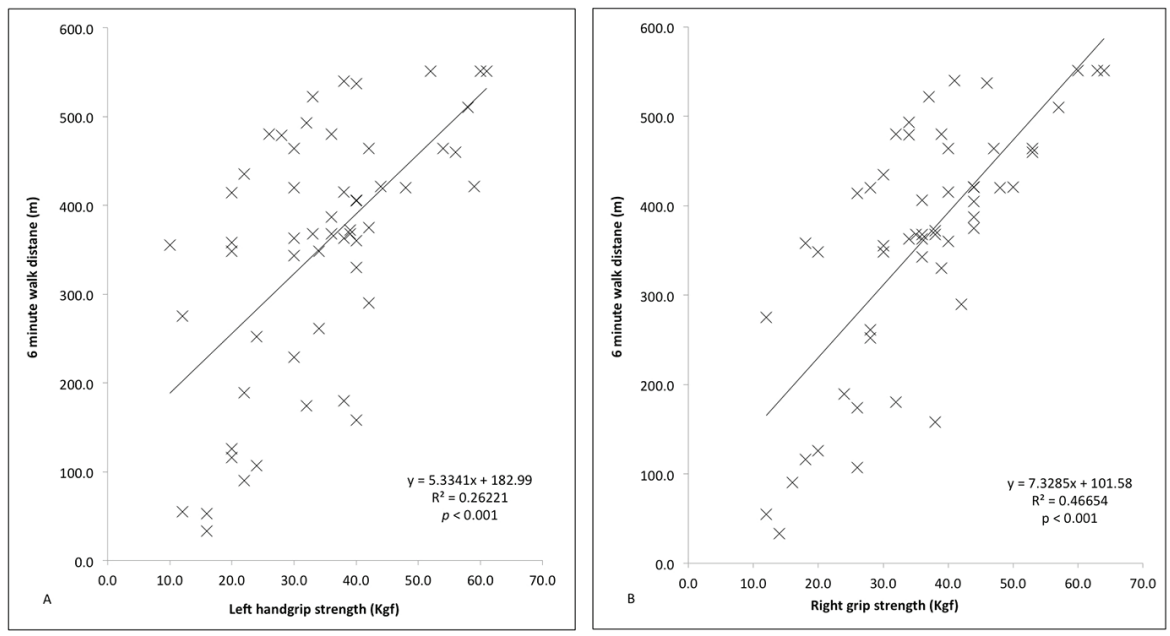

Figure 5. Correlation between handgrip strength (right and left) and 6-minute walk distance (6MWD) in the left (A) and right (B) hands both responders and non-responders, compared using Pearson's correlation coefficient.

measuring gross weight alone is fraught with error, as the HF condition leads to fluid retention and significant fluctuations in weight, but not muscle mass.

A possible confounder is serum albumin, an indicator of nutrition and associated with skeletal muscle performance [24-26]. Differences between the groups could reflect a more nutritionally replete/deplete cohort (as with HGS). However, serum albumin concentrations (mean and SD) were within normal limits (reference range $35-50 \mathrm{~g} / \mathrm{L}$ ) for all patients and no statistically significant difference was found during followup in either group (data not shown). Serum albumin is influenced by other co-morbidities such as liver and renal disease, all of which remained stable throughout the 12-month period of assessment. Furthermore, future research would be needed to ensure patients are consuming consistently 25 - $30 \mathrm{~g}$ high quality protein at breakfast, lunch and dinner, to provide sufficient protein to effectively stimulate muscle protein anabolism and adequately control for this extraneous variable [27].

Importantly, there were no statistically significant differences at baseline between the two groups. Although there were some non-significant differences, this is likely to be due to the small sample size. This cohort was a small but homogenous sample, but there were no ethnic minorities and only one woman and so it is difficult to translate the results to such populations. Whilst any improvement in HGS following a positive response to CRT implantation is correlation, not causation, there were no other significant clinical interventions during the 12 months follow-up.

Defining response to CRT is problematic and subjective, for this reason the authors decided that any responder must demonstrate improvement in four completely different markers at certain thresholds, widely used in the literature, but not collectively $[11,28-30]$. This was to ensure, as much as possible, that any response was true and not due to random variation, particularly in such a small patient population. Indeed, there was an improvement, albeit non-significant, in the MLWHFQ score in non-responders, but this is likely to be due to chance or placebo effect, especially as this was associated with no other response.

Taking into account both age and disease, this patient cohort has similar values of HGS to those reported in other CHF studies $[5,7]$. The only intervention study examining HGS and HF demonstrated an increase in HGS of $2 \mathrm{kgf}$ following a 3-month exercise programme [6]. The present CRT study demonstrates that similar gains at 12 months are found in CRT in patients who respond, on average $18 \%$ increase per hand. CRT allows patients to increase their own activity voluntarily, rather than as part of a specific exercise programme. It would be interesting to see if, as Belardinelli et al (2006) found with flow mediated dilatation (FMD) and endothelial dysfunction, further gains in HGS could be achieved by the adoption of an exercise programme and what, if any, effect this might have on other markers of response [31]. To date, there are no studies examining HGS in HF patients before and after routine cardiac rehabilitation. A recent study however demonstrated that exercise training in HF patients led to increases in muscle force and reductions in the levels of MuRF-1, a component of the ubiquitin-proteasome system involved in muscle proteolysis, which is increased in the skeletal muscle of patients with HF [32]. It would be interesting to see what impact positive response CRT also had on such a biomarker.

There were no clinical events during 12 months follow-up, such as hospitalizations or deaths in either group, but in larger trials with longer follow-up, it would be interesting to see if such improvements in HGS were correlated with reductions in adverse events such as morbidity and mortality.

\section{Conclusions}

This study demonstrates for the first time that there are significant improvements in the skeletal muscle performance of CRT responders, which correlates with 6MWD. This supports the notion that HGS is a simple, quick and inexpensive method of 
identifying responders to CRT. However, there are no significant differences in HGS between responders and non-responders to CRT at baseline, and so based on this study, it cannot be used to identify responders.

This improvement in HGS may reflect the fact that HF is not purely a single organ disease and so such measures before and after initiating HF therapies should be considered to corroborate improvement in symptoms and ensure this is not simply due to response bias, placebo or conformity. If larger studies demonstrated differences in HGS at baseline, the prospect of using a single simple test such as HGS to predict response to CRT remains a tantalizing possibility.

This was a small study in a homogenous population and so the findings will need replication in larger, diverse cohorts with such measurements at baseline and follow-up. Finally, further research is needed to investigate the mechanism between CRT and improved HGS.

\section{Acknowledgments}

This work would not have been possible without the help of Justin Adams and Debbie Wilkinson-Lill in the Echocardiography Department and Tracey Hughes and Cheryl Roberts in the Pulmonary Function Unit at the Northern General Hospital, Sheffield Teaching Hospitals NHS Trust, Sheffield.

\section{Funding}

This work was supported by a grant from the Engineering and Physical Sciences Research Council (EPSRC): Translating biomedical modelling into the heart of the clinic $(\mathrm{R} / 125661$ 11-1).

\section{References}

1. Hunt SA, Abraham WT, Chin MH, Feldman AM, Francis GS, Ganiats TG, Jessup M, et al. 2009 focused update incorporated into the ACC/AHA 2005 Guidelines for the Diagnosis and Management of Heart Failure in Adults: a report of the American College of Cardiology Foundation/American Heart Association Task Force on Practice Guidelines: developed in collaboration with the International Society for Heart and Lung Transplantation. Circulation. 2009;119(14):e391-479.

2. Coats AJ, Clark AL, Piepoli M, Volterrani M, Poole-Wilson PA. Symptoms and quality of life in heart failure: the muscle hypothesis. Br Heart J. 1994;72(2 Suppl):S36-39.

3. Izawa KP, Watanabe S, Osada N, Kasahara Y, Yokoyama H, Hiraki K, Morio Y, et al. Handgrip strength as a predictor of prognosis in Japanese patients with congestive heart failure. Eur J Cardiovasc Prev Rehabil. 2009;16(1):2127.

4. Castillo Martinez L, Colin Ramirez E, Orea Tejeda A, Asensio Lafuente E, Bernal Rosales LP, Rebollar Gonzalez V, Narvaez David R, et al. Bioelectrical imped- ance and strength measurements in patients with heart failure: comparison with functional class. Nutrition. 2007;23(5):412-418.

5. Izawa KP, Watanabe S, Yokoyama H, Hiraki K, Morio $\mathrm{Y}$, Oka K, Osada N, et al. Muscle strength in relation to disease severity in patients with congestive heart failure. Am J Phys Med Rehabil. 2007;86(11):893-900.

6. Gary RA, Cress ME, Higgins MK, Smith AL, Dunbar SB. Combined aerobic and resistance exercise program improves task performance in patients with heart failure. Arch Phys Med Rehabil. 2011;92(9):1371-1381.

7. Senden PJ, Sabelis LW, Zonderland ML, Hulzebos EH, Bol E, Mosterd WL. The effect of physical training on workload, upper leg muscle function and muscle areas in patients with chronic heart failure. Int J Cardiol. 2005;100(2):293-300.

8. Anker SD, Negassa A, Coats AJ, Afzal R, Poole-Wilson PA, Cohn JN, Yusuf S. Prognostic importance of weight loss in chronic heart failure and the effect of treatment with angiotensin-converting-enzyme inhibitors: an observational study. Lancet. 2003;361(9363):1077-1083.

9. Schellenbaum GD, Smith NL, Heckbert SR, Lumley T, Rea TD, Furberg CD, Lyles MF, et al. Weight loss, muscle strength, and angiotensin-converting enzyme inhibitors in older adults with congestive heart failure or hypertension. J Am Geriatr Soc. 2005;53(11):1996-2000.

10. Cleland JG, Daubert JC, Erdmann E, Freemantle N, Gras D, Kappenberger L, Tavazzi L. The effect of cardiac resynchronization on morbidity and mortality in heart failure. N Engl J Med. 2005;352(15):1539-1549.

11. Freemantle N, Tharmanathan P, Calvert MJ, Abraham WT, Ghosh J, Cleland JG. Cardiac resynchronisation for patients with heart failure due to left ventricular systolic dysfunction - a systematic review and meta-analysis. Eur J Heart Fail. 2006;8(4):433-440.

12. Mathiowetz V, Weber K, Volland G, Kashman N. Reliability and validity of grip and pinch strength evaluations. J Hand Surg Am. 1984;9(2):222-226.

13. Fess EE. Grip Strength. 2nd ed. Chicago: American Society of Hand Therapists; 1992.

14. Wilson JR, Martin JL, Schwartz D, Ferraro N. Exercise intolerance in patients with chronic heart failure: role of impaired nutritive flow to skeletal muscle. Circulation. 1984;69(6):1079-1087.

15. Mancini DM, Walter G, Reichek N, Lenkinski R, McCully KK, Mullen JL, Wilson JR. Contribution of skeletal muscle atrophy to exercise intolerance and altered muscle metabolism in heart failure. Circulation. 1992;85(4):1364-1373.

16. Opasich C, Ambrosino N, Felicetti G, Aquilani R, Pasini E, Bergitto D, Mazza A, et al. Heart failure-related myopathy. Clinical and pathophysiological insights. Eur Heart J. 1999;20(16):1191-1200.

17. Sullivan MJ, Green HJ, Cobb FR. Skeletal muscle biochemistry and histology in ambulatory patients with longterm heart failure. Circulation. 1990;81(2):518-527.

18. Mettauer B, Zoll J, Sanchez H, Lampert E, Ribera F, Veksler V, Bigard X, et al. Oxidative capacity of skeletal muscle in heart failure patients versus sedentary or active 
control subjects. J Am Coll Cardiol. 2001;38(4):947-954.

19. Hambrecht R, Fiehn E, Yu J, Niebauer J, Weigl C, Hilbrich L, Adams V, et al. Effects of endurance training on mitochondrial ultrastructure and fiber type distribution in skeletal muscle of patients with stable chronic heart failure. J Am Coll Cardiol. 1997;29(5):1067-1073.

20. Meyer FJ, Borst MM, Zugck C, Kirschke A, Schellberg D, Kubler W, Haass M. Respiratory muscle dysfunction in congestive heart failure: clinical correlation and prognostic significance. Circulation. 2001;103(17):21532158.

21. Fielding RA, Vellas B, Evans WJ, Bhasin S, Morley JE, Newman AB, Abellan van Kan G, et al. Sarcopenia: an undiagnosed condition in older adults. Current consensus definition: prevalence, etiology, and consequences. International working group on sarcopenia. J Am Med Dir Assoc. $2011 ; 12(4): 249-256$.

22. Fulster S, Tacke M, Sandek A, Ebner N, Tschope C, Doehner W, Anker SD, et al. Muscle wasting in patients with chronic heart failure: results from the studies investigating co-morbidities aggravating heart failure (SICAHF). Eur Heart J. 2013;34(7):512-519.

23. Clark BC, Manini TM. Sarcopenia =/= dynapenia. J Gerontol A Biol Sci Med Sci. 2008;63(8):829-834.

24. Bistrian BR, Blackburn GL, Vitale J, Cochran D, Naylor J. Prevalence of malnutrition in general medical patients. JAMA. 1976;235(15):1567-1570.

25. Baumgartner RN, Koehler KM, Romero L, Garry PJ. Serum albumin is associated with skeletal muscle in elderly men and women. Am J Clin Nutr. 1996;64(4):552-558.

26. Wang AY, Sea MM, Ho ZS, Lui SF, Li PK, Woo J. Evaluation of handgrip strength as a nutritional marker and prognostic indicator in peritoneal dialysis patients. Am J Clin Nutr. 2005;81(1):79-86.

27. Bosaeus I, Rothenberg E. Nutrition and physical activity for the prevention and treatment of age-related sarcopenia. Proc Nutr Soc. 2016;75(2):174-180.

28. Diaz-Infante E, Mont L, Leal J, Garcia-Bolao I, Fernandez-Lozano I, Hernandez-Madrid A, Perez-Castellano N, et al. Predictors of lack of response to resynchronization therapy. Am J Cardiol. 2005;95(12):1436-1440.

29. Moss AJ, Hall WJ, Cannom DS, Klein H, Brown MW, Daubert JP, Estes NA, 3rd, et al. Cardiac-resynchronization therapy for the prevention of heart-failure events. $\mathrm{N}$ Engl J Med. 2009;361(14):1329-1338.

30. Hoogslag GE, Hoke U, Thijssen J, Auger D, Marsan NA, Wolterbeek R, Holman ER, et al. Clinical, echocardiographic, and neurohormonal response to cardiac resynchronization therapy: are they interchangeable? Pacing Clin Electrophysiol. 2013;36(11):1391-1401.

31. Belardinelli R, Capestro F, Misiani A, Scipione P, Georgiou D. Moderate exercise training improves functional capacity, quality of life, and endothelium-dependent vasodilation in chronic heart failure patients with implantable cardioverter defibrillators and cardiac resynchronization therapy. Eur J Cardiovasc Prev Rehabil. 2006;13(5):818825.

32. Gielen S, Sandri M, Kozarez I, Kratzsch J, Teupser D, Thiery J, Erbs S, et al. Exercise training attenuates MuRF1 expression in the skeletal muscle of patients with chronic heart failure independent of age: the randomized Leipzig Exercise Intervention in Chronic Heart Failure and Aging catabolism study. Circulation. 2012;125(22):27162727. 